\title{
A Proximal-Point Outer Approximation Algorithm
}

\author{
Massimo De Mauri ${ }^{\dagger \ddagger}$, Joris Gillis ${ }^{\ddagger}$, Jan Swevers ${ }^{\ddagger}$ and Goele Pipeleers ${ }^{\ddagger}$ * \\ July 15,2020
}

\begin{abstract}
Many engineering and scientific applications, e.g. resource allocation, control of hybrid systems, scheduling, etc., require the solution of Mixed-Integer Non-Linear Problems (MINLPs). Problems of such class combine the high computational burden arising from considering discrete variables with the complexity of non-linear functions. As a consequence, the development of algorithms able to efficiently solve medium-large MINLPs is still an interesting field of research.

In the last decades, several approaches to tackle MINLPs have been developed. Some of such approaches, usually defined as exact methods, aim at finding a globally optimal solution for a given MINLP at expense of a long execution time, while others, generally defined as heuristics, aim at discovering suboptimal feasible solutions in the shortest time possible. Among the various proposed paradigms, Outer Approximation (OA) and Feasibility Pump (FP), respectively as exact method and as heuristic, deserve a special mention for the number of relevant publications and successful implementations related to them.

In this paper we present a new exact method for convex mixed-integer non-linear programming called Proximal Outer Approximation (POA). POA blends the fundamental ideas behind FP into the general OA scheme that attepts to yield faster and more robust convergence with respect to OA while retaining the good performances in terms of fast generation of feasible solutions of FP.
\end{abstract}

\section{Introduction}

Mixed-Integer non-linear programming is a branch of numerical optimization that deals with optimization problems in which some of the variables are only allowed to take values within a certain discrete set and either or both the objective-function and the constraints are non-linear. Mixed-Integer Non-Linear Problems (MINLPs) are very commonly encountered in scientific and engineering applications and numerous efforts have been spent on the development of efficient solution algorithms for them ([1],[2],[4],[8],[11]). However, MINLPs are still particularly computationally expensive to solve.

In practice, MINLPs are much harder to solve than both Non-Linear Problems (NLPs) and Mixed-Integer Linear Problems (MILPs). Therefore, Duran and Grossmann in 1986 [1] proposed an algorithm, called Outer Approximation (OA), that finds an optimal point for the given convex MINLP via the solution of an alternating sequence of MILPs and NLPs $([3],[4],[12],[13])$. After almost forty years, the algorithmic ideas of the original OA method are still at the core of some of the most competitive MINLP solution approaches. Supporting evidence for that can be found on recent survey from Kronqvist et al. [10]. The survey shows that, on the selected benchmark, the most robust and performative solvers to date are those based on some form of outer approximation, as for example SHOT ([7],[9]) and AOA ([8]).

However, the theoretical complexity of $\mathrm{OA}$ is exponential in the number of discrete variables and the practical solvability of a problem instance depends, among other factors, on how rapidly the algorithm is capable of identifying feasible points for it. In fact, OA derives its convergence from the construction and the iterative refinement of a linear approximation of the non-linear constraint set of the MINLP to solve. The refinements are obtained by adding to the current linear approximation new linearizations of the original constraint-set. Using feasible points it is possible to obtain stronger linearizations ([5]), therefore, better refinements and quicker convergence. Consequently, it is common in practice to deploy heuristic methods to rapidly retrieve feasible points in the early stages of MINLPs optimizations $([20],[26],[27])$. For example, in [29] the authors propose to prepend to the OA iterations some iterations of the widely used heuristics for MINLPs named Feasibility Pump (FP) ([21],[15],[20]). Furthermore, in this setting, it is also important to deploy heuristics that are capable of rapidly retrieve close-tooptimal points for a given MINLP $([25],[28])$. In facts, a close-to-optimal feasible point for a problem provides a tight upper bound on the globally optimal objective-value of the problem and, in turn, such upper bound can be

\footnotetext{
*Acknowledgment: This work has been carried out within the framework of KU Leuven - BOF PFV/10/002 Centre of Excellence: Optimization in Engineering (OPTEC) and Flanders Make ICON: Physical and control co-design of electromechanical drivetrains for machines and vehicles. Flanders Make is the Flemish strategic research centre for the manufacturing industry.

${ }^{\dagger}$ Email: massimo.demauri@kuleuven.be

${ }_{\ddagger}^{\ddagger}$ Affiliation: MECO Research Team, Department Mechanical Engineering, KU Leuven and Flanders Make - DMMS_M, Leuven, Belgium.
} 
used to speed-up the convergence. In this direction goes the concept of objective-FP $([20],[21],[25])$ from which the present work takes some inspiration.

In this paper a new algorithm for the solution of convex MINLPs, called Proximal Outer Approximation (POA), is presented. Differently from the algorithm presented in [29], our solution scheme follows an integrated approach. The underlying idea is to incorporate some of the fundamental concepts of FP into the general OA scheme. Specifically, the NLP step of POA closely resembles the one of FP while its MILP step is an adaptive trade-off between the more feasibility focused MILP step of FP and the more optimality focused MILP step of OA. Such operation results into an optimization scheme that breaks through the distinction between main scheme and supporting heuristic yielding fast convergence properties while retaining good performances in terms of rapid retrieval of feasible solutions. It is important to note that the presented work does not stand isolated in literature. For instance, in [5] the authors propose a modification of the MILP step of OA that is similar to the one hereby proposed, in [6] the concept of inner-approximation for separable MINLPs is introduced in order to ease the task of providing feasible integer guesses and, analogously, the algorithm presented in [11] selects integer guesses close to the center of the current linear outer approximation.

The performed numerical experiments show that the theoretical advantages of the proposed method result, on the examined benchmark, in shorter solution times, less iterations and a larger number of problem instances successfully solved within the time limits with respect to classical OA.

The remainder of the paper is organized in five sections. In the first section we introduce the class of problems that the proposed algorithm solves. The second section provides the reader with a brief introduction to OA and FP. In the third section, our proposed algorithmic approach is described. Finally, in the last two sections, the supporting computational results are shown and some conclusions drawn.

\section{Problem Statement}

Consider the following MINLP:

$$
\begin{array}{ll}
\min _{x, y} & C_{x}^{T} x+C_{y}^{T} y \\
\text { s.t. } & g(x, y) \leq 0 \\
& x \in \mathbb{R}^{n_{x}}, y \in \mathbb{Z}^{n_{y}}
\end{array}
$$

where $C_{x} \in \mathbb{R}^{n_{x}}$ and $C_{y} \in \mathbb{R}^{n_{y}}$ collect the coefficients for the linear objective and $g(x, y): \mathbb{R}^{n_{x}} \times \mathbb{R}^{n_{y}} \rightarrow \mathbb{R}^{n_{g}}$ is a vector-valued possibly non-linear function of its arguments.

It is easy to see that, perhaps performing an epigraph reformulation on the objective-function, any MINLP can

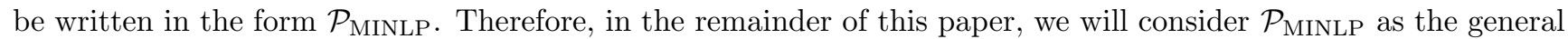
form of a MINLP.

A point $(\tilde{x}, \tilde{y})$ is "constraint-feasible" for $\mathcal{P}_{\text {MINLP }}$ if $g(\tilde{x}, \tilde{y}) \leq 0$, while, a point $(\bar{x}, \bar{y})$ that satisfies the condition $\bar{y} \in \mathbb{Z}^{n_{y}}$ is said "integer-feasible" for $\mathcal{P}_{\text {MINLP. A point }}(x, y)$ is feasible for $\mathcal{P}_{\text {MINLP }}$ if it is simultaneously constraintand integer- feasible for $\mathcal{P}_{\text {MINLP }}$ and it is said infeasible otherwise. Further, let the set $Y$ be defined as follows:

$$
Y:=\left\{y \in \mathbb{Z}^{n_{y}} \text { s.t. } \exists x \in \mathbb{R}^{n_{x}}: g(x, y) \leq 0\right\}
$$

With a slight abuse of terminology, we are going to call a discrete assignment $\bar{y}$ feasible if $\bar{y} \in Y$ and infeasible otherwise.

Throughout this paper, we assume the following:

(A-1) The constraints set $g(x, y) \leq 0$ contains the bounding constraints $l_{y} \leq y \leq u_{y}$ where $\left\|l_{y}\right\|_{\infty},\left\|u_{y}\right\|_{\infty}<\infty$

(A-2) The function $g$ is continuously differentiable and jointly convex in $x$ and $y$.

(A-3) A constraint qualification, such to guarantee the satisfaction of the first order KKT conditions, holds at the solution of every NLP obtained from $\mathcal{P}_{\text {MINLP }}$ by fixing the value of the variables $y$ to a discrete assignment $\bar{y} \in Y$.

Given an iterative optimization algorithm, let the incumbent solution $I$ at iteration $k$ be defined as the best feasible point found by the algorithm during all the previous iterations.

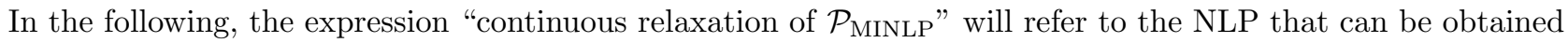
from $\mathcal{P}_{\text {MINLP }}$ by replacing the condition: $y \in \mathbb{Z}^{n_{y}}$, with the condition: $y \in \mathbb{R}^{n_{y}}$. 


\section{Previous Work}

\subsection{The Outer Approximation Algorithm}

$\mathrm{OA}$ is a multi-step iterative procedure that generates an alternating sequence of integer-feasible and constraintfeasible guesses for $(x, y)$ which are guaranteed to converge together to a globally optimal point for the MINLP at hand.

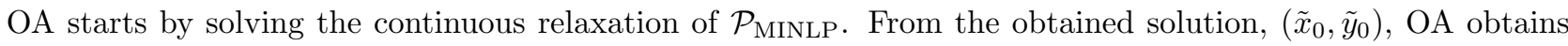
an integer-feasible (but possibly constraint infeasible) guess $\bar{y}_{0}$ on the value of the discrete variables of the MINLP via a rounding step. Then, the following quantities are defined:

- A lower bound on the optimal value for $\mathcal{P}_{\text {MINLP: }}$

$$
V^{\mathrm{LB}}:=-\infty
$$

- An upper bound on the optimal value for $\mathcal{P}_{\text {MINLP: }}$

$$
V^{\mathrm{UB}}:=\infty
$$

- An initial set of linear constraints:

$$
\begin{aligned}
L_{x, 0}:=\frac{\partial g}{\partial x}\left(\tilde{x}_{0}, \tilde{y}_{0}\right) \\
L_{y, 0}:=\frac{\partial g}{\partial y}\left(\tilde{x}_{0}, \tilde{y}_{0}\right) \\
l_{0}:=g\left(\tilde{x}_{0}, \tilde{y}_{0}\right)-\frac{\partial g}{\partial x, y} g\left(\tilde{x}_{0}, \tilde{y}_{0}\right)^{T}\left[\begin{array}{c}
\tilde{x}_{0} \\
\tilde{y}_{0}
\end{array}\right]
\end{aligned}
$$

Then, at each iteration $k$, OA attempts at solving the following NLP:

$$
\begin{aligned}
\tilde{x}_{k}:=\underset{x}{\operatorname{argmin}} & C_{x}^{T} x+C_{y}^{T} \bar{y}_{k-1} \\
\text { s.t. } & g\left(x, \bar{y}_{k-1}\right) \leq 0 \\
& x \in \mathbb{R}^{n_{x}}
\end{aligned}
$$

If the above problem is feasible, i.e. $\bar{y}_{k-1} \in Y$, then $\left(\tilde{x}_{k}, \bar{y}_{k-1}\right)$ is a feasible point for $\mathcal{P}_{\text {MINLP yielding an upper }}$ bound on the optimal objective-value for $\mathcal{P}_{\text {MINLP }}$. Consequently, in case:

$$
V^{\mathrm{UB}}>C_{x}^{T} \tilde{x}_{k}+C_{y}^{T} \bar{y}_{k-1}
$$

the upper bound, $V^{\mathrm{UB}}$, and the incumbent solution, $I$, can be updated as follows:

$$
\begin{array}{ll}
V^{\mathrm{UB}} & :=C_{x}^{T} \tilde{x}_{k}+C_{y}^{T} \bar{y}_{k-1} \\
I & :=\left(\tilde{x}_{k}, \bar{y}_{k-1}\right)
\end{array}
$$

However, frequently, $\mathcal{P}_{1}$ results infeasible. In that case, $\tilde{x}_{k}$ is defined as the solution of an NLP of the following form:

$$
\begin{aligned}
\tilde{x}_{k}:=\underset{x, s}{\operatorname{argmin}} & w^{T} s \\
\text { s.t. } & g\left(x, \bar{y}_{k-1}\right) \leq s \\
& s \geq 0 \\
& x \in \mathbb{R}^{n_{x}}, s \in \mathbb{R}^{n_{g}}
\end{aligned}
$$

where, $s$ is a vector of "slack" variables and $w$ is a vector of non-negative weights. The idea behind $\mathcal{P}_{2}$ is to find a $\tilde{x}_{k}$ that, in some sense, minimizes the constraint-infeasibility of $\left(\tilde{x}_{k}, \bar{y}_{k-1}\right)$.

Whether $\mathcal{P}_{1}$ resulted feasible or not, the constraint function $g$ is linearized via a first order Taylor expansion around $\left(\tilde{x}_{k}, \bar{y}_{k-1}\right)$ and the resulting linear approximations are added to the current set of linear approximations as follows:

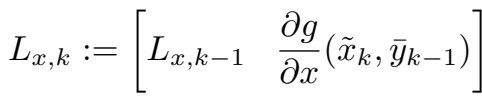

$$
\begin{aligned}
& L_{y, k}:=\left[\begin{array}{ll}
L_{y, k-1} & \left.\frac{\partial g}{\partial y}\left(\tilde{x}_{k}, \bar{y}_{k-1}\right)\right]
\end{array}\right.
\end{aligned}
$$

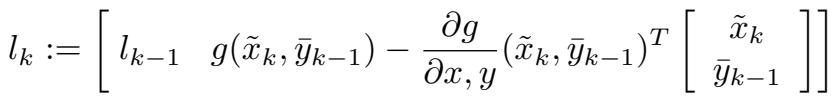


Next, a new integer-feasible guess is found by solving the following MILP:

$$
\begin{aligned}
& \left(\bar{x}_{k}, \bar{y}_{k}\right):=\underset{x, y}{\operatorname{argmin}} C_{x}^{T} x+C_{y}^{T} y \\
& \text { s.t. } \quad L_{x, k}^{T} x+L_{y, k}^{T} y+l_{k}^{T} \leq 0 \\
& x \in \mathbb{R}^{n_{x}}, y \in \mathbb{Z}^{n_{y}}
\end{aligned}
$$

Thanks to the convexity assumption (A-2), at each iteration $k$, the linear function:

$$
g\left(\tilde{x}_{k}, \bar{y}_{k-1}\right)+\frac{\partial g}{\partial x}\left(\tilde{x}_{k}, \bar{y}_{k-1}\right)^{T}\left(x-\tilde{x}_{k}\right)+\frac{\partial g}{\partial y}\left(\tilde{x}_{k}, \bar{y}_{k-1}\right)^{T}\left(y-\bar{y}_{k-1}\right)
$$

under-estimates $g(x, y)$. Therefore, the feasible space defined from $L_{x, k}^{T} x+L_{y, k}^{T} y+l_{k}^{T} \leq 0$ contains the feasible space defined from $g(x, y) \leq 0$. Consequently, the optimal value of $\mathcal{P}_{3}$ yields a lower bound on the optimal value of $\mathcal{P}_{\text {MINLP. }}$ Then, $V^{\mathrm{LB}}$ can be updated as follows:

$$
V^{\mathrm{LB}}:=\max \left\{C_{x}^{T} \bar{x}_{k}+C_{y}^{T} \bar{y}_{k}, V^{\mathrm{LB}}\right\}
$$

Now, if the following condition is met $^{1}$ :

$$
V^{\mathrm{UB}}-V^{\mathrm{LB}} \leq \epsilon_{\mathrm{abs}} \quad \text { or } \quad \frac{V^{\mathrm{UB}}-V^{\mathrm{LB}}}{\delta+\left|V^{\mathrm{UB}}\right|} \leq \epsilon_{\mathrm{rel}} \quad: \delta>0
$$

The algorithm stops as $I$ is guaranteed to be an optimal point (up to the required tolerance) for $\mathcal{P}_{\text {MINLP. Otherwise, }}$ OA proceeds with the next iteration.

Figure 1: Schematic Representation of Outer Approximation

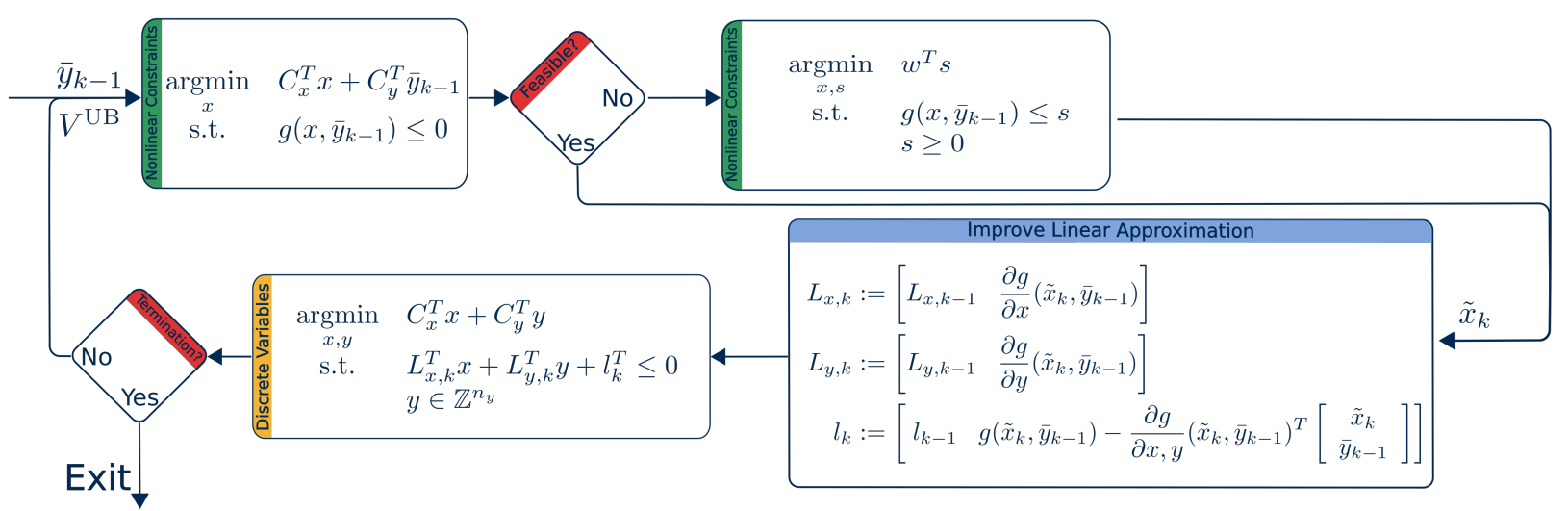

\subsection{The Non-Linear Feasibility Pump}

Similarly to OA, the non-linear FP generates an alternating sequence of integer-feasible and constraint-feasible guesses for $(x, y)$. Differently from OA, however, the FP scheme guarantees that the two sequences will converge to a feasible assignment for $(x, y)$, disregarding optimality.

FP starts by computing $\left(\tilde{x}_{0}, \tilde{y}_{0}\right)$ as the solution of the continuous relaxation of $\mathcal{P}_{\text {MINLP. Next, an initial }}$ linear approximation for the non-linear constraints set $g$ is obtained as in (1). Then, at each iteration, a new integer-feasible guess for $(x, y)$ is generated by solving the following MILP:

$$
\begin{aligned}
\left(\bar{x}_{k}, \bar{y}_{k}\right):=\underset{x, y}{\operatorname{argmin}} & \left\|y-\tilde{y}_{k-1}\right\|_{1} \\
\text { s.t. } & L_{x, k}^{T} x+L_{y, k}^{T} y+l_{k}^{T} \leq 0 \\
& x \in \mathbb{R}^{n_{x}}, y \in \mathbb{Z}^{n_{y}}
\end{aligned}
$$

Next, a constraint-feasible guess for $(x, y)$ is found via the solution of the following NLP:

$$
\begin{aligned}
\left(\tilde{x}_{k}, \tilde{y}_{k}\right):=\underset{x, y}{\operatorname{argmin}} & \frac{1}{2}\left\|y-\bar{y}_{k}\right\|_{2}^{2} \\
\text { s.t. } & g(x, y) \leq 0 \\
& x \in \mathbb{R}^{n_{x}}, y \in \mathbb{R}^{n_{y}}
\end{aligned}
$$

\footnotetext{
${ }^{1}$ Using the Cplex definition of the relative optimality gap
} 
If $\bar{y}_{k} \in Y$ then $\tilde{y}_{k}=\bar{y}_{k}$ and the point $\left(\tilde{x}_{k}, \tilde{y}_{k}\right)$ is feasible for $\mathcal{P}_{\text {MINLP }}$, therefore, the algorithm stops. Otherwise, a new set of linear approximations, is obtained as follows:

$$
\begin{aligned}
& L_{x, k+1}:=\left[\begin{array}{ll}
L_{x, k} & \left.\frac{\partial g}{\partial x}\left(\tilde{x}_{k}, \tilde{y}_{k}\right)\right]
\end{array}\right. \\
& L_{y, k+1}:=\left[\begin{array}{ll}
L_{y, k} & \left.\frac{\partial g}{\partial y}\left(\tilde{x}_{k}, \tilde{y}_{k}\right)\right]
\end{array}\right.
\end{aligned}
$$

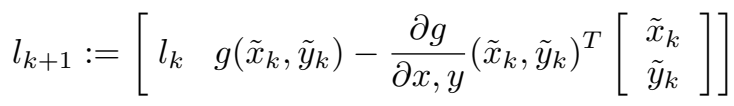

and a new iteration starts.

Note that $\mathcal{P}_{4}$ can be interpreted as an advanced rounding technique that takes into account the current linear

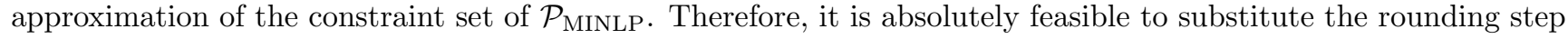
at the start-up of OA with few iterations of FP as for example in [29].

$\mathcal{P}_{4}$ can also be interpreted as an operator that projects the current constraint-feasible solution into the intersection between the domain of the discrete variables and the feasible space defined by the current set of approximating linear constraints. Similarly, $\mathcal{P}_{5}$ can be interpreted as projecting the current integer-feasible solution into the feasible space of $\mathcal{P}_{\text {MINLP. }}$. This has led some researchers to interpret FP as an "alternating direction method" ([17],[30]).

FP has proven to be a very effective primal heuristic for MINLPs but the feasible points it finds are usually of limited quality in terms of optimality. This is due to the fact that the original objective-function is considered exclusively during the generation of the first discrete assignment.

Figure 2: Schematic Representation of Non-Linear Feasibility Pump

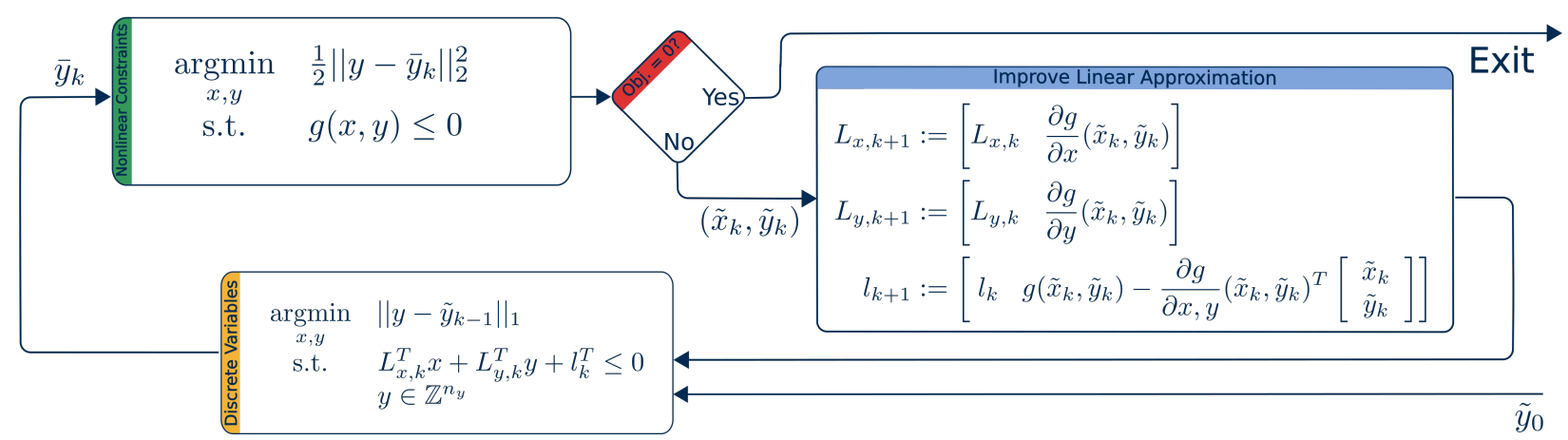

\section{Proximal Outer Approximation}

\subsection{Structure of POA}

Just like OA, POA starts by computing $\left(\tilde{x}_{0}, \tilde{y}_{0}\right)$ as the solution of the continuous relaxation of $\mathcal{P}_{\text {MINLP }}$ it defines $\bar{y}_{0}$ rounding $\tilde{y}_{0}$. Furthermore, POA defines the same initial set of linear approximations, $\left(L_{x, 0}, L_{y, 0}, l_{0}\right)$, that OA defines.

Then, the following bounds are defined:

- A lower bound on the optimal value for $\mathcal{P}_{\text {MINLP: }}$

$$
V^{\mathrm{LB}}:=C_{x}^{T} \tilde{x}_{0}+C_{y}^{T} \tilde{y}_{0}
$$

- An upper bound on the optimal value for $\mathcal{P}_{\mathrm{MINLP}}$ :

$$
V^{\mathrm{UB}}:=\infty
$$

Next, a constraint feasible guess for $(x, y)$ is obtained by solving the following FP-like $\mathrm{NLP}^{1}$ :

$$
\begin{aligned}
\left(\tilde{x}_{k}, \tilde{y}_{k}\right):=\underset{x, y}{\operatorname{argmin}} & \frac{1}{2}\left\|y-\bar{y}_{k-1}\right\|_{2}^{2} \\
\text { s.t. } & C_{x}^{T} x+C_{y}^{T} y \leq V^{\mathrm{UB}}-\epsilon_{\mathrm{abs}} \\
& g(x, y) \leq 0 \\
& x \in \mathbb{R}^{n_{x}}, y \in \mathbb{R}^{n_{y}}
\end{aligned}
$$


where $\epsilon_{\text {abs }}$ it is the user provided absolute optimality tolerance ${ }^{2}$.

Now, we can distinguish two cases:

1. The optimal objective of $\mathcal{P}_{6}$ is greater than zero or, equivalently, $\tilde{y}_{k} \neq \bar{y}_{k-1}$.

$\Longrightarrow \bar{y}_{k-1}$ is infeasible or there exist no point $\left(x, \bar{y}_{k-1}\right)$ such that $g\left(x, \bar{y}_{k-1}\right) \leq 0$ whose objective-value

is strictly lower than the provided upper bound $V^{\mathrm{UB}}$.

2. The optimal objective of $\mathcal{P}_{6}$ is equal to zero or, equivalently, $\tilde{y}_{k}=\bar{y}_{k-1}$.

$\Longrightarrow \quad\left(\tilde{x}_{k}, \bar{y}_{k-1}\right)$ is a feasible point yielding a better objective-value than the one yielded by the incumbent solution.

In the second case, POA attempts to refine $\tilde{x}_{k}$ via the solution of problem $\mathcal{P}_{1}$, just like OA (with the difference that, at this point, the feasibility of $\mathcal{P}_{1}$ is guaranteed). Consequently, $V^{\mathrm{UB}}$ and the incumbent solution is updated as follows:

$$
\begin{array}{ll}
V^{\mathrm{UB}} & :=C_{x}^{T} \tilde{x}_{k}+C_{y}^{T} \tilde{y}_{k} \\
I & :=\left(\tilde{x}_{k}, \tilde{y}_{k}\right)
\end{array}
$$

Then, a new set of linearizations is defined as follows:

$$
\begin{aligned}
& L_{x, k}:=\left[\begin{array}{ll}
L_{x, k-1} & \left.\frac{\partial g}{\partial x}\left(\tilde{x}_{k}, \tilde{y}_{k}\right)\right]
\end{array}\right. \\
& L_{y, k}:=\left[\begin{array}{ll}
L_{y, k-1} & \left.\frac{\partial g}{\partial y}\left(\tilde{x}_{k}, \tilde{y}_{k}\right)\right]
\end{array}\right.
\end{aligned}
$$

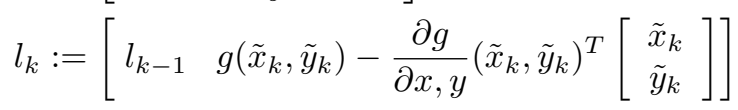

Next, a new integer-feasible guess is generated via the solution of the following MILP:

$$
\begin{aligned}
\left(\bar{x}_{k}, \bar{y}_{k}\right):=\underset{x, y}{\operatorname{argmin}} & f_{k}(x, y) \\
\text { s.t. } & C_{x}^{T} x+C_{y}^{T} y \leq V^{\mathrm{UB}}-\epsilon_{\mathrm{abs}} \\
& L_{x, k}^{T} x+L_{y, k}^{T} y+l_{k}^{T} \leq 0 \\
& x \in \mathbb{R}^{n_{x}}, y \in \mathbb{Z}^{n_{y}}
\end{aligned}
$$

where:

$$
f_{k}(x, y):=\alpha_{k} \frac{\left(C_{x}^{T} x+C_{y}^{T} y\right)}{N_{o, k}}+\left(1-\alpha_{k}\right) \frac{\left\|y-\tilde{y}_{k-1}\right\|_{1}}{N_{d, k}}
$$

with $N_{o, k}$ and $N_{d, k}$ normalization parameters and $\alpha_{k} \in[0,1]$ is used to determine a convex combination of the two normalized objectives.

If $\alpha$ is set to one and $\mathcal{P}_{7}$ results feasible, $\left(\bar{x}_{k}, \bar{y}_{k}\right)$ yields a lower bound for the globally optimal objective-value of $\mathcal{P}_{\text {MINLP. }}$. Therefore, in such case, the lower bound $V^{\mathrm{LB}}$ can be updated as follows:

$$
V^{\mathrm{LB}}:=C_{x}^{T} \bar{x}_{k}+C_{y}^{T} \bar{y}_{k}
$$

Eventually, POA stops whenever $\mathcal{P}_{7}$ the following condition is met:

$$
\mathcal{P}_{7} \text { results infeasible } \quad \text { or } \quad V^{\mathrm{UB}}-V^{\mathrm{LB}} \leq \epsilon_{\mathrm{abs}} \quad \text { or } \quad \frac{V^{\mathrm{UB}}-V^{\mathrm{LB}}}{10^{-10}+\left|V^{\mathrm{UB}}\right|} \leq \epsilon_{\mathrm{rel}}
$$

In any case, if at the time of termination, if an incumbent solution is present, such is an optimal point (up to the defined tolerances) for $\mathcal{P}_{\text {MINLP }}$, otherwise, $\mathcal{P}_{\text {MINLP }}$ is proven infeasible. A schematic representation of the POA algorithm can be found in fig. 3 .

It is important to note that the first constraint in $\mathcal{P}_{7}$ has two functions:

1. It prevents POA from discovering the same integer assignment twice (so that the finite termination of the algorithm can be guaranteed).

\footnotetext{
${ }^{2}$ It is common practice, while solving MINLPs, to define positive absolute and relative optimality gap tolerances. Such quantities are used to stop the algorithm whenever the desired solution accuracy is met. Moreover, setting the optimality gap tolerances to zero in a OA-like algorithm might lead to numerical difficulties as, normally, the NLP solvers can guarantee constraint satisfaction and optimality only up to a certain tolerance.

In POA, $\epsilon_{\text {abs }}$ is used in the MILP and NLP formulation to implement a strict inequality on the objective-value. As a positive side effect, the algorithm is forced to ignore all the feasible assignments that have no chance of improving the current best objective of at least the defined absolute tolerance.
} 
Figure 3: Schematic Representation of POA

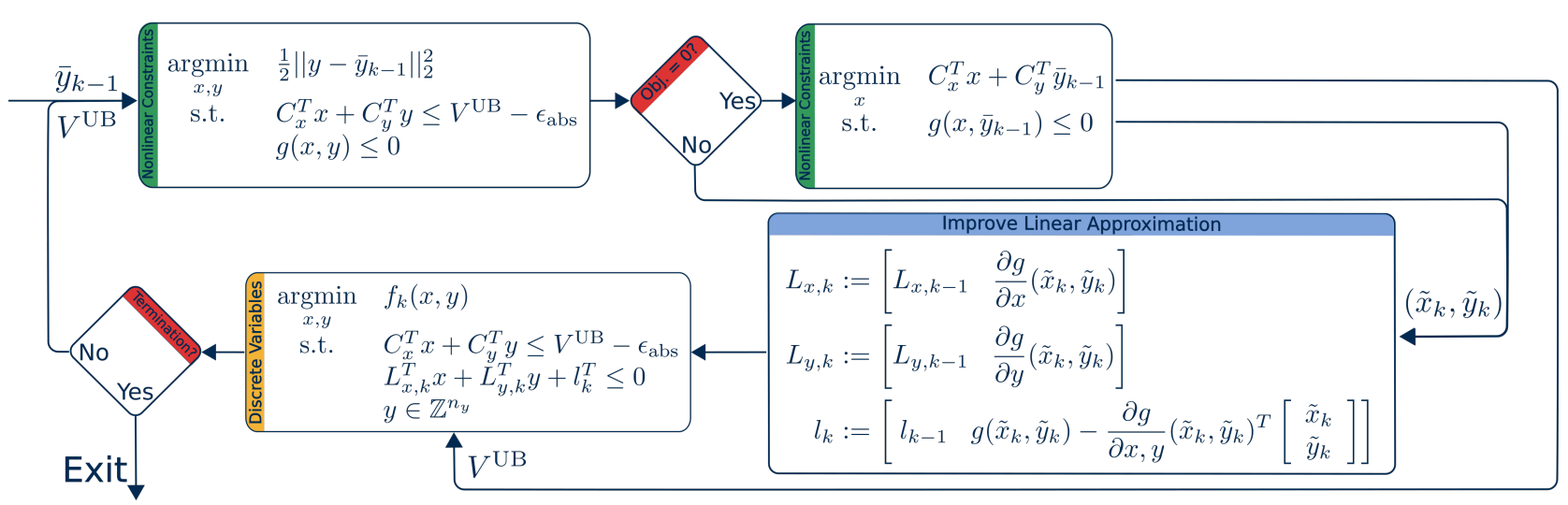

2. Forces the MILP to ignore clearly suboptimal integer assignments also in case $\alpha_{k}<1$.

Moreover, the convexity assumption (A-1) guarantees that the feasible space defined by $L_{x, k}^{T} x+L_{y, k}^{T} y+l_{k}^{T} \leq$ 0 strictly contains the one defined by $g(x, y) \leq 0$. Consequently, the possible infeasibility of $\mathcal{P}_{7}$ implies the nonexistence of any assignment for $(x, y)$ simultaneously respecting the non-linear constraints of $\mathcal{P}_{\text {MINLP }}$ and providing an objective-value lower than the current upper bound.

If, instead, $\mathcal{P}_{7}$ is feasible and $\alpha_{k}=1$, then the optimal objective-value of $\mathcal{P}_{7}$ is a lower bound on the optimal objective-value of $\mathcal{P}_{\text {MINLP }}$, and:

$$
V^{\mathrm{LB}}:=C_{x}^{T} \bar{x}_{k}+C_{y}^{T} \bar{y}_{k}
$$

\subsection{POA compared to OA}

Although the general structure of POA is very similar to the one of OA, the two algorithms differ in two important aspects.

\subsubsection{Discrete assignment guessing}

OA selects, as guess, always the discrete assignment providing the best objective-value in the MILP stage. Therefore, when the available set of linearizations is a poor approximation of the original non-linear set of constraints, OA tends to generate guesses which are far from the non-linear feasible space and thus scarcely informative. Conversely, POA tries to balance the relaxed objective-value of assignments with their closeness to the non-linear feasible space. Consequently, POA generates guesses which are closer to the non-linear feasible space and therefore more informative in the sense that they generally provide tighter outer approximations.

\subsubsection{Linear approximations generation}

In case of infeasibility of a discrete assignment guess $y$, OA solves problem $\mathcal{P}_{2}$ in order to find a continuous assignment $x$ such that $(x, y)$ minimizes a certain infeasibility measure. The result of this operation is a linearization point that lies outside of the feasible space of $\mathcal{P}_{\text {MINLP }}$ and whose location depends heavily on the weighting vector $W$. As shown in [7], via a numerical experiment, generating linearizations for a non-linear constraint set from outside its feasible space might lead to loose linear approximations. Consequently, OA might occasionally perform more iterations than necessary and a bad scaling of the non-linear constraints set can severely hinder the convergence of the algorithm. Differently, in the same circumstances, POA finds a linearization point which lies right at the boundary of the feasible space of $\mathcal{P}_{\text {MINLP }}$ via the projection step $\mathcal{P}_{6}$. Moreover, at the linearization point found by POA all components of $g(x, y) \leq 0$ making $y$ infeasible are active. As a result, independently from the scaling of $g(x, y)$, POA generates tighter linear approximations with respect to OA.

\subsection{Convergence and Exactness of POA}

\subsubsection{POA converges in finite time}

The bounds defined on $y$ are assumed finite. Therefore, to prove the finite convergence of POA is enough to prove that, denoting with $\bar{y}_{k}$ the discrete assignment found by the MILP at the $k$-th iteration $\left(\operatorname{MILP}_{k}\right)$, the following condition holds:

$$
\bar{y}_{k+h} \neq \bar{y}_{k-1}: \forall h \in \mathbb{N}
$$


Since no constraint is ever removed from the linearizations set, the above condition is guaranteed to hold if the linear approximations generated during the $k$-th iteration suffice to make any point $\left(x, \bar{y}_{k-1}\right): x \in \mathbb{R}^{n_{x}}$ infeasible for $\mathrm{MILP}_{k}$.

Now, let us distinguish two cases, the case where $\bar{y}_{k-1}$ is feasible, or $\bar{y}_{k-1} \in Y$, and the case where it is not, or $\bar{y}_{k-1} \notin Y$. In both cases, we will prove that, for all $x$ such that $C_{x}^{T} x+C_{y}^{T} \bar{y}_{k-1}<V^{\mathrm{UB}},\left(x, \bar{y}_{k-1}\right)$ violates the first order Taylor approximation of at least one of the components of $g(x, y) \leq 0$ which are strictly active at the solution of either $\mathcal{P}_{1}$ or $\mathcal{P}_{6}$.

Lemma 1: Let $\bar{y}_{k-1} \in Y$ and let $\tilde{x}_{k}$ be the solution of $\mathcal{P}_{1}$ then: for all $x \in \mathbb{R}^{n_{x}}$ with $C_{x}^{T} x<C_{x}^{T} \tilde{x}_{k}$ there exist $i \in\left\{1, \ldots, n_{y}\right\}$ such that the constraint $g_{i}(x, y)$ is strictly active at $\left(\tilde{x}_{k}, \bar{y}_{k-1}\right)$ and it holds:

$$
\frac{\partial g_{i}}{\partial x}\left(\tilde{x}_{k}, \bar{y}_{k-1}\right)^{T}\left(x-\tilde{x}_{k}\right)>0
$$

Proof: From the KKT conditions applied to $\mathcal{P}_{1}$, we obtain:

$$
\left\{\begin{array}{c}
\sum_{i} \mu_{i} \frac{\partial g_{i}}{\partial x}\left(\tilde{x}_{k}, \bar{y}_{k-1}\right)=-C_{x} \\
\mu_{i} \geq 0 \quad \forall i \in\left\{1, \ldots, n_{g}\right\}
\end{array}\right.
$$

Therefore, for $x \in \mathbb{R}^{n_{x}}$ such that $C_{x}^{T} x<C_{x}^{T} \tilde{x}_{k}$, we have:

$$
\sum_{i} \mu_{i} \frac{\partial g_{i}}{\partial x}\left(\tilde{x}_{k}, \bar{y}_{k-1}\right)^{T}\left(x-\tilde{x}_{k}\right)=-C_{x}^{T}\left(x-\tilde{x}_{k}\right)>0
$$

Then, the desired result follows directly from the non-negativity of the Lagrangian multipliers.

Lemma 2: Let $\bar{y}_{k-1} \notin Y$ and let $\left(\tilde{x}_{k}, \tilde{y}_{k}\right)$ be the solution of $\mathcal{P}_{6}$. Then, there exist $i \in\left\{1, \ldots, n_{y}\right\}$ such that the constraint $g_{i}(x, y)$ is strictly active at $\left(\tilde{x}_{k}, \tilde{y}_{k}\right)$ and, $\forall x \in \mathbb{R}^{n_{x}}$, holds:

$$
\frac{\partial g_{i}}{\partial x}\left(\tilde{x}_{k}, \tilde{y}_{k}\right)^{T}\left(x-\tilde{x}_{k}\right)+\frac{\partial g_{i}}{\partial y}\left(\tilde{x}_{k}, \tilde{y}_{k}\right)^{T}\left(\bar{y}_{k-1}-\tilde{y}_{k}\right)>0
$$

Proof: Denoting as $D(x, y)$ the function $\frac{1}{2}\left\|y-\bar{y}_{k-1}\right\|_{2}^{2}$, we get:

$$
\left\{\begin{array}{l}
\frac{\partial D}{\partial x}\left(\tilde{x}_{k}, \tilde{y}_{k}\right)=0 \\
\frac{\partial D}{\partial y}\left(\tilde{x}_{k}, \tilde{y}_{k}\right)=\tilde{y}_{k}-\bar{y}_{k-1}
\end{array}\right.
$$

moreover, from the KKT conditions we obtain that:

$$
\left\{\begin{array}{l}
-\frac{\partial D}{\partial x}\left(\tilde{x}_{k}, \tilde{y}_{k}\right)=\sum_{i} \mu_{i} \frac{\partial g_{i}}{\partial x}\left(\tilde{x}_{k}, \tilde{y}_{k}\right) \\
-\frac{\partial D}{\partial y}\left(\tilde{x}_{k}, \tilde{y}_{k}\right)=\sum_{i} \mu_{i} \frac{\partial g_{i}}{\partial y}\left(\tilde{x}_{k}, \tilde{y}_{k}\right) \\
\mu_{i} \geq 0 \forall i \in\left\{1, \ldots, n_{g}\right\}
\end{array}\right.
$$

Therefore, for any assignment $\left(x, \bar{y}_{k-1}\right)$, with $x \in \mathbb{R}^{n_{x}}$, we have:

$$
\begin{gathered}
\sum_{i} \mu_{i}\left(\frac{\partial g_{i}}{\partial x}\left(\tilde{x}_{k}, \tilde{y}_{k}\right)^{T}\left(x-\tilde{x}_{k}\right)+\frac{\partial g_{i}}{\partial y}\left(\tilde{x}_{k}, \tilde{y}_{k}\right)^{T}\left(\bar{y}_{k-1}-\tilde{y}_{k}\right)\right)= \\
-\left(\frac{\partial D}{\partial x}\left(\tilde{x}_{k}, \tilde{y}_{k}\right)^{T}\left(x-\tilde{x}_{k}\right)+\frac{\partial D}{\partial y}\left(\tilde{x}_{k}, \tilde{y}_{k}\right)^{T}\left(\bar{y}_{k-1}-\tilde{y}_{k}\right)\right)= \\
\left(\tilde{y}_{k}-\bar{y}_{k-1}\right)^{T}\left(\tilde{y}_{k}-\bar{y}_{k-1}\right)>0
\end{gathered}
$$

Finally, the desired result follows from the non-negativity of the Lagrangian multipliers.

Now, let $i$ be an index such that $g_{i}(x, y)<=0$ is strictly active at the solution of either $\mathcal{P}_{1}$ or $\mathcal{P}_{6}$. Possibly renaming $\bar{y}_{k-1}$ to $\tilde{y}_{k}$, we have: $g_{i}\left(\tilde{x}_{k}, \tilde{y}_{k}\right)=0$. Therefore, taking the first order Taylor expansion of $g_{i}(x, y)<=0$ around $\left(\tilde{x}_{k}, \tilde{y}_{k}\right)$, we get the following linear constraint:

$$
\frac{\partial g_{i}}{\partial x}\left(\tilde{x}_{k}, \tilde{y}_{k}\right)^{T}\left(x-\tilde{x}_{k}\right)+\frac{\partial g_{i}}{\partial y}\left(\tilde{x}_{k}, \tilde{y}_{k}\right)^{T}\left(y-\tilde{y}_{k}\right) \leq 0
$$


The two lemmas above prove that for any point $\left(x, \bar{y}_{k-1}\right): x \in \mathbb{R}^{n_{x}}$ it exists one index $i^{*}$ such that $\left(x, \bar{y}_{k-1}\right)$ violates either the above linear constraint or the upper-bound defined in (4). Therefore, thanks to (5), the discrete assignment $\bar{y}_{k-1}$ is infeasible for every $\operatorname{MILP}_{k+h}: h \in \mathbb{R}$. As a consequence, $\bar{y}_{k-1}$ cannot be discovered twice and, thanks to the boundedness of the set $Y$, this guarantees the finite convergence of POA.

For the sake of completeness, we would like to refer the interested reader to [3] and [5] where similar properties are proven in slightly different contexts.

\subsubsection{POA converges to an optimal point for the MINLP}

POA stops whenever MILP $_{k}$, for some $k$, results infeasible, i.e., whenever:

$$
\nexists(x, y) \in \mathbb{R}^{n_{x}} \times \mathbb{Z}^{n_{y}} \text { s.t. }\left\{\begin{array}{l}
L_{x, k}^{T} x+L_{y, k}^{T} y+l_{k}^{T} \leq 0 \\
C_{x}^{T} x+C_{y}^{T} y<V^{\mathrm{UB}}
\end{array}\right.
$$

Now, given the convexity of the non-linear constraints set $g(x, y) \leq 0$, we have that the linear constraints $L_{x, k}^{T} x+$ $L_{y, k}^{T} y+l_{k}^{T} \leq 0$ form a relaxation of $g(x, y) \leq 0$. Then, it follows:

$$
\nexists(x, y) \in \mathbb{R}^{n_{x}} \times \mathbb{Z}^{n_{y}} \text { s.t. }\left\{\begin{array}{l}
g(x, y) \leq 0 \\
C_{x}^{T} x+C_{y}^{T} y<V^{\mathrm{UB}}
\end{array}\right.
$$

Therefore:

- If $V^{\mathrm{UB}}=\infty$, there is no incumbent solution and the original MINLP is infeasible.

- If $V^{\mathrm{UB}}$ is finite, the incumbent solution, whose objective-value is $V^{\mathrm{UB}}$, is globally optimal for the original MINLP.

\subsection{Setting the parameters}

At each iteration, POA solves a MILP in order to find a new guess on the optimal discrete assignment for the MINLP. The MILP objective is a combination of the MINLP objective and a $L_{1}$ distance term. To each term a different relative importance is given depending on the value of the parameter $\alpha_{k}$. The idea behind such structure is to look for new discrete assignments trading off between their potential objective-value and their chances of constraint-feasibility.

In fact, when the current linear approximations set is a poor approximation of the original non-linear constraints set, it is beneficial to focus more on constraint-feasibility chances in order to rapidly retrieve good solutions. On the other hand, whenever the generated linearizations form a good approximation of the nonlinear feasible space, focusing on minimal objective-value leads to the globally optimal point in a shorter amount of time.

In the next paragraphs, an attempt to put some basis for the development of a general rule for the $\alpha_{k}$ parameter will be presented.

\subsubsection{Normalization Constants}

First of all, we need to minimize the impact of the scaling of the MINLP objective on its importance in the MILP. Therefore, we aim to find a couple of iteration dependent constants, $N_{o, k}$ and $N_{d, k}$, such that the normalized terms $\frac{C_{x}^{T} x+C_{y}^{T} y}{N_{o, k}}$ and $\frac{\left\|y-\tilde{y}_{k-1}\right\|_{1}}{N_{d, k}}$ have, approximately, the same magnitude. A classical solution to this problem consists in normalizing the two terms using their values at the extremes of the pareto-optimal curve arising from varying the value of $\alpha_{k} \in[0,1]$. Clearly, such approach is impractical as it requires the solution of two additional MILPs, one with $\alpha_{k}=1$ and the other with $\alpha_{k}=0$, at each iteration. A cheaper approximate method consists in setting:

$$
\begin{aligned}
& N_{o, k}=C_{x}^{T}\left(\tilde{x}_{k-1}-\tilde{x}_{0}\right)+C_{y}^{T}\left(\tilde{y}_{k-1}-\tilde{y}_{0}\right) \\
& N_{d, k}=\left\|\tilde{y}_{0}-\tilde{y}_{k-1}\right\|_{1}
\end{aligned}
$$

This approximation performs well in practice and requires almost no additional computational load.

\subsubsection{Setting $\alpha_{k}$}

It is not straightforward to a priori determine a general rule for the $\alpha_{k}$ values. In this section we will limit our self to present some of the expertise on the matter gained during the development phase of the presented work.

First of all, we need to define a measurable quantity to monitor in order to assess the quality of the linear approximations set at each iteration. Lets, for the moment, denote such quantity with $q_{k}$ and assume that $q_{k}$ 
tends to zero as the conservativism of the linear constraint set $\left(L_{x, k}, L_{y, k}, l_{k}\right)$ is iteratively reduced. Then, it is necessary to map $q_{k}$ into a suitable value of $\alpha_{k}$. In order to do so, we exploited the following algorithm depending on two parameters $(\tau, \lambda)$ :

$$
\begin{aligned}
Q_{k} & := \begin{cases}0 & \text { if } k=0 \\
(\lambda-1) Q_{k-1}+\lambda q_{k} & \text { otherwise }\end{cases} \\
\tilde{\alpha}_{k} & :=\frac{\tau}{\tau+Q_{k}} \\
\alpha_{k} & := \begin{cases}1 & \text { if } \tilde{\alpha}_{k} \geq 0.99 \\
\tilde{\alpha}_{k} & \text { otherwise }\end{cases}
\end{aligned}
$$

where $Q_{k}$ is some sort of integrated measurement or running average with $\lambda$ as damping parameter and $\tau$ is used as scaling factor for $Q_{k}$ in the soft thresholding function $\frac{1}{1+x}$. It is important to note that the hard thresholding in the last step of $\alpha_{\mathrm{alg}}(\tau, \lambda)$ serves the vital purpose of allowing POA to set $\alpha_{k}$ to exactly one when $q_{k}$ approaches zero. In facts, when $\alpha_{k}=1$ problem $\mathcal{P}_{7}$ provides a lower bound on the optimal objective-value for $\mathcal{P}_{\text {MINLP }}$ and such lower bound can be used to trigger the termination of the algorithm.

Coming back to the definition of $q_{k}$, a number metrics have been tested out. For each of the metrics, several tests have been performed varying the values of the two parameters in $\alpha_{\mathrm{alg}}(\tau, \lambda)$. Each test consisted in solving a number of problem instances taken from MINLPLib [34] and storing the yielded performances for each of the considered instances. At the end of the testing phase the most promising approach resulted the one following two:

1. $L_{1}$ averaged distance along the discrete directions between the solution of $\mathcal{P}_{7}$ and its projection onto the non-linear feasible space:

$$
q_{k}=\frac{\left\|\bar{y}_{k-1}-\tilde{y}_{k}\right\|_{1}}{n_{y}}
$$

2. Average violation of the non-linear constraints at the solution of $\mathcal{P}_{7}$ :

$$
q_{k}=\frac{\left\|\max \left(g\left(\bar{x}_{k-1}, \bar{y}_{k-1}\right), 0\right)\right\|_{1}}{n_{g}}
$$

Consequently, in the next section only the two above metrics will be considered to showcase the possible advantages in using the hereby presented POA algorithm.

\section{Numerical Evaluation}

This section presents a comparison between the performances of four different algorithms applied to a number of problem instances taken from MINLPLib [34]. The four considered algorithms are:

1. OA: a basic Outer Approximation implementation algorithm as described in the second section of this paper.

2. $\mathrm{FP}+\mathrm{OA}$ : the algorithm performs Feasibility Pump iterations until a first feasible solution is found then it continues applying Outer Approximation until finding a solution or exceeding the time limit.

3. $\operatorname{POA}_{1}(1,1 / 3)$ : Proximal Outer Approximation where alpha is computed according to algorithm $\alpha_{\mathrm{alg}}(\tau, \lambda)$ with $q_{k}$ computed as in $(12)$ and $(\tau:=1, \lambda:=1 / 3)$

4. $\mathrm{POA}_{2}(100,1 / 3)$ : Proximal Outer Approximation where alpha is computed according to algorithm $\alpha_{\mathrm{alg}}(\tau, \lambda)$ with $q_{k}$ computed as in $(13)$ and $(\tau:=100, \lambda:=1 / 3)$

The tests hereby presented were performed on a personal computer equipped with a $3.7 \mathrm{GHz}$ octa-core processor (AMD Ryzen 7 2700X) and $16 \mathrm{~Gb}$ of RAM, selecting some instances from MINLPLib ([34]) according to the following rules:

1. Mixed-integer/mixed-boolean variable set and non-linear constraints set and/or objective.

2. Proven convex instances with known globally optimal objective (found by at least 3 solvers).

3. Not relaxations of other problems in the set (to the authors knowledge).

4. Having a ".nl" representation (14 instances excluded for this reason). 
For each problem instance and each algorithm a maximum of 300 seconds of computation was allowed, the primal and the integer tolerances where set to $10^{-6}$ while the absolute and relative gap tolerances were set to $10^{-4}$. In order to obtain a fair comparison all the algorithms share the same base code and general sub-solver settings. All the tested algorithms use Cplex v12.9 ([33]) and Ipopt v3.12.11([31]). In the appendix at the end of this paper a table can be found showing the computational time and the number iterations each algorithm needs to obtain the global optimal solution for each problem instance. A problem instance is considered successfully solved by an algorithm if the algorithm runtime does not excess 303 seconds $(+1 \%$ of the imposed limit to account for possible non-instantaneous terminations) and the obtained objective-value falls within a relative or absolute gap of $10^{-3}$ from the known globally optimal objective-value of the instance. In the table, all the unsuccessful runs are denoted with the symbol - in place of the number of iterations and the runtime. ${ }^{3}$

In the remainder of this section, the global performances of the tested algorithms are summarized and compared with the aid of two metrics: number of instances solved and the shifted geometric mean of both the computational times and the numbers of iterations. In the following, $\operatorname{SGM}(q, s)$ will indicate the shifted geometric mean of the quantity $q$ given the shift $s$ :

$$
\operatorname{SGM}(q, s):=\left(\prod_{i=1}^{n_{q}}\left(s+q_{i}\right)\right)^{1 / n_{q}}-s: q \in \mathbb{R}^{n_{q}}, s \in[0, \infty)
$$

In the coming evaluation, we consider only those problem instances where all the algorithms resulted successful. The shift used for each quantity was selected in order to limit the impact of very easy problems (solved in less of one second and in one or two iterations) in the final comparison. Table 1 summarizes the collected data.

\begin{tabular}{|c||r|r|r|r|}
\hline Metric & OA & $\mathrm{FP}+\mathrm{OA}$ & $\mathrm{POA}_{1}(1,1 / 3)$ & $\mathrm{POA}_{2}(100,1 / 3)$ \\
\hline \# of solved instances & 210 & 210 & 213 & 212 \\
$S G M$ (times, 0.5) & 1.83 & 1.74 & 1.66 & 1.62 \\
$S G M($ \# iterations, 1.5) & 6.95 & 7.46 & 6.21 & 5.80 \\
\hline
\end{tabular}

Table 1: Absolute performances of the analyzed algorithms (full dataset).

According to the averages presented in Table 1, it is possible to compare each algorithm against the classical OA. The result of such comparison can be found in Table 2.

Evaluating the complexity of each instance using as reference the performances of the algorithm that performed the best on them, table 3 shows the comparison obtained considering only the most time-demanding $25 \%$ of the instances while table 4 considers only the most iteration-intesive $25 \%$ of the instances.

\begin{tabular}{|c||r|r|r|}
\hline Performances VS OA & FP + OA & $\mathrm{POA}_{1}(1,1 / 3)$ & $\mathrm{POA}_{2}(100,1 / 3)$ \\
\hline Complexity (time) & $-4.66 \%$ & $-9.38 \%$ & $-11.57 \%$ \\
Complexity (\# iterations) & $+7.48 \%$ & $-10.65 \%$ & $-16.49 \%$ \\
\hline
\end{tabular}

Table 2: Performance comparison versus classical OA (full dataset).

\begin{tabular}{|c||r|r|r|}
\hline Performances VS OA & FP + OA & $\mathrm{POA}_{1}(1,1 / 3)$ & $\mathrm{POA}_{2}(100,1 / 3)$ \\
\hline Complexity (time) & $-7.18 \%$ & $-11.00 \%$ & $-8.31 \%$ \\
Complexity (\# iterations) & $+10.81 \%$ & $-13.64 \%$ & $-16.24 \%$ \\
\hline
\end{tabular}

Table 3: Performance comparison versus classical OA (time-demanding instances).

\begin{tabular}{|c||r|r|r|}
\hline Performances VS OA & FP + OA & $\mathrm{POA}_{1}(1,1 / 3)$ & $\mathrm{POA}_{2}(100,1 / 3)$ \\
\hline Complexity (time) & $-1.90 \%$ & $-41.17 \%$ & $-42.85 \%$ \\
Complexity (\# iterations) & $+2.69 \%$ & $-46.35 \%$ & $-48.55 \%$ \\
\hline
\end{tabular}

Table 4: Performance comparison versus classical OA (iteration-intensive instances).

On the one hand, Table 2 shows how, especially on time-demanding problems, prepending an FP run to the OA iterations is likely to reduce the average time needed to find the solution, explaining the popularity of such approach. On the other hand, Table 4 shows that the advantages of FP tend to be nullified over the more iterationintensive problems. This is probably due to the fact that, for those problems, the effort necessary to find the first feasible solution is overshadowed by the large number of iterations required in order to close the optimality gaps.

Regarding POA, we can see that both the tested versions maintain a good performance edge over the classical algorithm in all analyzed scenarios, with $\mathrm{POA}_{2}(100,1 / 3)$ being more effective than $\operatorname{POA}_{1}(1,1 / 3)$ in general but slightly less effective on the more time-intensive instances. The most noteworthy finding comes from Table 4 . The table shows how the presented algorithm is capable of providing very large computational savings in those 
problems where, due to the high level of non-linearity in the constraint set, the construction of an accurate enough linear relaxation requires a large number of linearization steps.

\section{Conclusions}

In this paper we presented a new solution algorithm for convex mixed-integer non-linear programming named Proximal Outer Approximation. The proposed algorithm combines ideas and properties of Outer Approximation and Non-Linear Feasibility Pump to construct an integrated iterative solution scheme.

The aim of this work was to create an Outer Approximation scheme with improved generation of approximating linear constraints and better discrete assignment candidates selection. The performed computational experiments have shown the advantages of the new scheme over both the classical Outer Approximation and Outer Approximation warm-started by Feasibility Pump and suggested that, through a careful selection of parameters, an ulterior performance improvement is attainable.

In this paper, we have found $\mathrm{POA}_{2}(100,1 / 3)$ having the best average and peak performance among all the analyzed algorithms. However, this does not exclude the possibility that for different classes of problems different parameter choices could result more efficient.

\footnotetext{
${ }^{3}$ The appendix contains also the full timing and number of iterations data relative to Bonmin. The data shows how, in terms of number of iterations, our test code is capable of matching the performances of a state-of-the-art OA implementation. However, the data shows also that Bonmin has a good margin of advantage over our test code in terms of execution times. This is due to the fact our test code was built as a proof of concept and, while our code was implemented in Python, Bonmin enjoys a fairly optimized C implementation.
} 


\begin{tabular}{|c|c|c|c|c|c|c|c|c|c|c|c|}
\hline \multicolumn{2}{|r|}{ Examples } & \multicolumn{2}{|c|}{ Bonmin } & \multicolumn{2}{|c|}{$\mathrm{OA}$} & \multicolumn{2}{|c|}{$\mathrm{OA}+\mathrm{FP}$} & \multicolumn{2}{|c|}{$\mathrm{POA}_{1}(1,1 / 3)$} & \multicolumn{2}{|c|}{$\mathrm{POA}_{2}(100,1 / 3)$} \\
\hline $\mathrm{Id}$ & Name & \#It. & Time & \#It. & Time & \#It. & Time & \#It. & Time & \#It. & Time \\
\hline 1 & batch & - & - & - & - & - & - & - & - & - & - \\
\hline 2 & batch0812 & - & - & - & - & - & - & - & - & - & - \\
\hline 3 & batchdes & - & - & - & - & - & - & - & - & - & - \\
\hline 4 & batchs101006m & - & - & - & - & - & - & - & - & - & - \\
\hline 5 & batchs $121208 \mathrm{~m}$ & - & - & - & - & - & - & - & - & - & - \\
\hline 6 & batchs $151208 \mathrm{~m}$ & - & - & - & - & - & - & - & - & - & - \\
\hline 7 & batchs $201210 \mathrm{~m}$ & - & - & - & - & - & - & - & - & - & - \\
\hline 8 & clay0203h & 9 & 0.56 & 8 & 0.55 & 8 & 0.48 & 9 & 0.49 & 11 & 0.69 \\
\hline 9 & clay0203hfsg & 8 & 0.49 & 6 & 0.39 & 7 & 0.38 & 6 & 0.34 & 6 & 0.35 \\
\hline 10 & clay $0203 \mathrm{~m}$ & 9 & 0.35 & 8 & 0.33 & 9 & 0.35 & 11 & 0.5 & 10 & 0.51 \\
\hline 11 & clay0204h & 3 & 0.55 & 3 & 0.35 & 1 & 0.13 & 6 & 0.52 & 4 & 0.41 \\
\hline 12 & clay0204hfsg & 3 & 0.45 & 3 & 0.32 & 4 & 0.31 & 4 & 0.78 & 4 & 0.47 \\
\hline 13 & clay0204m & 3 & 0.15 & 4 & 0.24 & 5 & 0.22 & 5 & 0.26 & 7 & 0.41 \\
\hline 14 & clay0205h & 4 & 5.83 & 5 & 2.85 & 4 & 2.47 & 5 & 3.74 & 8 & 6.11 \\
\hline 15 & clay0205m & 6 & 1.34 & 6 & 1.3 & 9 & 1.78 & 9 & 1.81 & 8 & 1.65 \\
\hline 16 & clay0303h & 9 & 0.95 & 7 & 0.69 & 7 & 0.77 & 11 & 0.67 & 13 & 0.81 \\
\hline 17 & clay0303hfsg & 6 & 0.44 & 5 & 0.46 & 6 & 0.42 & 7 & 0.45 & 7 & 0.50 \\
\hline 18 & clay0303m & 10 & 0.5 & 8 & 0.38 & 13 & 0.5 & 9 & 0.43 & 11 & 0.57 \\
\hline 19 & clay0304h & 12 & 2.32 & 15 & 2.84 & 11 & 2.03 & 14 & 1.97 & 17 & 2.12 \\
\hline 20 & clay0304hfsg & 9 & 1.63 & 10 & 1.72 & 11 & 1.47 & 8 & 1.02 & 10 & 1.41 \\
\hline 21 & clay0304m & 18 & 1.58 & 17 & 1.33 & 21 & 1.44 & 16 & 1.5 & 21 & 2.02 \\
\hline 22 & clay0305h & 6 & 7.62 & 6 & 3.32 & 5 & 2.76 & 9 & 5.87 & 8 & 2.68 \\
\hline 23 & clay $0305 \mathrm{~m}$ & 6 & 1.55 & 7 & 1.66 & 10 & 2.12 & 9 & 2.47 & 11 & 2.19 \\
\hline 24 & cvxnonsep_normcon20 & 413 & 9.58 & 1 & 0.02 & 1 & 0.03 & 1 & 0.02 & 1 & 0.02 \\
\hline 25 & cvxnonsep_normcon30 & 1088 & 58.21 & 727 & 61.95 & 70 & 1.49 & 186 & 15.24 & 3 & 0.08 \\
\hline 26 & cvxnonsep_normcon 40 & 1876 & 120.45 & - & - & - & - & - & - & 3 & 0.07 \\
\hline 27 & cvxnonsep_nsig20 & 309 & 6.56 & 266 & 8.73 & 266 & 8.65 & 65 & 2.32 & 66 & 2.13 \\
\hline 28 & cvxnonsep_nsig30 & - & - & 1 & 0.03 & 1 & 0.05 & 1 & 0.03 & 1 & 0.04 \\
\hline 29 & cvxnonsep_nsig40 & - & - & 690 & 43.27 & 693 & 43.69 & 686 & 109.77 & 93 & 3.33 \\
\hline 30 & cvxnonsep_pcon20 & - & - & 56 & 1.91 & 59 & 2.24 & 30 & 0.87 & 26 & 0.76 \\
\hline 31 & cvxnonsep_pcon30 & - & - & 142 & 7.55 & 158 & 7.23 & 77 & 2.9 & 65 & 2.19 \\
\hline 32 & cvxnonsep_pcon40 & - & - & 317 & 21.5 & 292 & 19.82 & 167 & 8.43 & 160 & 6.44 \\
\hline 33 & cvxnonsep_psig20 & 531 & 11.12 & 1 & 0.02 & 1 & 0.02 & 1 & 0.02 & 1 & 0.02 \\
\hline 34 & cvxnonsep_psig30 & 704 & 40.28 & 630 & 31.6 & 600 & 31.1 & 177 & 11.48 & 152 & 5.66 \\
\hline 35 & cvxnonsep_psig40 & - & - & - & - & - & - & - & - & - & - \\
\hline 36 & enpro48pb & - & - & - & - & - & - & - & - & - & - \\
\hline 37 & enpro56pb & - & - & - & - & - & - & - & - & - & - \\
\hline 38 & ex1223 & 3 & 0.06 & 3 & 0.06 & 3 & 0.07 & 4 & 0.12 & 3 & 0.07 \\
\hline 39 & ex1223a & 1 & 0.04 & 1 & 0.03 & 1 & 0.02 & 1 & 0.02 & 1 & 0.02 \\
\hline 40 & ex1223b & 3 & 0.05 & 4 & 0.1 & 4 & 0.09 & 6 & 0.16 & 3 & 0.10 \\
\hline 41 & ex4 & 2 & 0.11 & 3 & 0.14 & 3 & 0.14 & 3 & 0.18 & 5 & 0.30 \\
\hline 42 & fac1 & 3 & 0.07 & - & - & - & - & 3 & 0.79 & 24 & 1.25 \\
\hline 43 & fac2 & 6 & 1.52 & - & - & - & - & 5 & 0.3 & 49 & 1.60 \\
\hline 44 & flay02h & 2 & 0.09 & 3 & 0.18 & 3 & 0.64 & 3 & 0.15 & 3 & 0.15 \\
\hline 45 & flay $02 \mathrm{~m}$ & 2 & 0.06 & 3 & 0.56 & 2 & 0.57 & 4 & 0.15 & 5 & 0.21 \\
\hline 46 & flay03h & 8 & 0.31 & 9 & 0.6 & 8 & 1.17 & 9 & 0.71 & 9 & 0.91 \\
\hline 47 & flay03m & 8 & 0.19 & 9 & 0.4 & 9 & 0.36 & 9 & 1.21 & 9 & 1.17 \\
\hline 48 & flay04h & 23 & 4.69 & 22 & 4.36 & 24 & 6.12 & 22 & 5.75 & 21 & 4.86 \\
\hline 49 & flay04m & 20 & 2.01 & 21 & 1.88 & 22 & 2.15 & 25 & 2.42 & 24 & 2.34 \\
\hline 50 & flay05h & - & - & 57 & 265.25 & 73 & 281.65 & 38 & 278.95 & 38 & 208.16 \\
\hline 51 & flay $05 \mathrm{~m}$ & - & - & 40 & 32.01 & 36 & 35.8 & 38 & 45.93 & 37 & 40.42 \\
\hline 52 & fo7 & 3 & 5.39 & 3 & 9.9 & 5 & 13.88 & 4 & 10.54 & 3 & 9.22 \\
\hline 53 & fo7_2 & 2 & 3.08 & 2 & 2.36 & 3 & 2.57 & 2 & 2.26 & 2 & 2.70 \\
\hline 54 & fo7_ar2_1 & 2 & 2.91 & 3 & 4.71 & 4 & 3.08 & 4 & 3.87 & 3 & 4.70 \\
\hline 55 & fo7_ar25_1 & 5 & 5.57 & 6 & 10.17 & 6 & 10.06 & 6 & 9.07 & 6 & 10.62 \\
\hline 56 & fo7_ar3_1 & 3 & 5.1 & 4 & 7.92 & 4 & 5.52 & 4 & 10.27 & 4 & 10.08 \\
\hline 57 & fo7_ar4_1 & 2 & 3.05 & 3 & 4.14 & 5 & 4.48 & 3 & 4.87 & 3 & 5.04 \\
\hline 58 & fo7_ar5_1 & 1 & 1.8 & 2 & 2.12 & 1 & 0.81 & 2 & 2.32 & 2 & 2.27 \\
\hline 59 & fo 8 & 2 & 7.09 & 3 & 17.1 & 4 & 11.08 & 2 & 10.26 & 2 & 10.18 \\
\hline 60 & fo8_ar2_1 & 2 & 12.68 & 4 & 41.04 & 4 & 13.51 & 4 & 26.72 & 4 & 38.24 \\
\hline 61 & fo8_ar25_1 & 3 & 15.37 & 4 & 26.92 & 7 & 36.77 & 4 & 28.16 & 4 & 35.07 \\
\hline 62 & fo8_ar3_1 & 1 & 1.99 & 2 & 3.29 & 4 & 3.22 & 2 & 2.9 & 3 & 4.43 \\
\hline 63 & fo8_ar4_1 & 1 & 4.21 & 2 & 3.8 & 3 & 3.01 & 2 & 4.18 & 2 & 4.24 \\
\hline 64 & fo8_ar5_1 & 1 & 4.18 & 2 & 8.74 & 4 & 8.04 & 3 & 9.52 & 3 & 9.37 \\
\hline 65 & fo9 & - & - & 3 & 45.08 & 4 & 53.2 & 3 & 46.3 & 3 & 45.43 \\
\hline 66 & fo9_ar3_1 & 1 & 4.75 & 2 & 3.92 & 3 & 6.03 & 2 & 7.37 & 2 & 5.32 \\
\hline 67 & fo9_ar4_1 & 1 & 3.53 & 2 & 7.68 & 4 & 11.45 & 2 & 10.31 & 2 & 9.91 \\
\hline 68 & fo9_ar5_1 & 3 & 23.05 & 4 & 24.87 & 4 & 26.17 & 4 & 40.77 & 4 & 40.78 \\
\hline 69 & jit1 & 5 & 0.31 & 1 & 0.02 & - & - & 1 & 0.02 & 1 & 0.02 \\
\hline 70 & $\mathrm{~m} 3$ & 1 & 0.04 & 1 & 0.03 & 1 & 0.04 & 1 & 0.04 & 1 & 0.06 \\
\hline 71 & $\mathrm{~m} 6$ & 2 & 0.19 & 2 & 0.11 & 4 & 0.14 & 2 & 0.19 & 2 & 0.13 \\
\hline 72 & $\mathrm{~m} 7$ & 1 & 0.26 & 2 & 0.18 & 3 & 0.22 & 2 & 0.23 & 2 & 0.26 \\
\hline
\end{tabular}




\begin{tabular}{|c|c|c|c|c|c|c|c|c|c|c|c|}
\hline \multicolumn{2}{|r|}{ Examples } & \multicolumn{2}{|c|}{ Bonmin } & \multicolumn{2}{|c|}{$\mathrm{OA}$} & \multicolumn{2}{|c|}{$\mathrm{OA}+\mathrm{FP}$} & $\mathrm{POA}$ & $(1,1 / 3)$ & $\mathrm{POA}$ & $00,1 / 3)$ \\
\hline Id & Name & \#It. & Time & \#It. & Time & \#It. & Time & \#It. & Time & \#It. & Time \\
\hline 73 & m7_ar2_1 & 1 & 0.35 & 2 & 0.54 & 3 & 0.62 & 2 & 0.62 & 2 & 0.53 \\
\hline 74 & m7_ar25_1 & 1 & 0.21 & 2 & 0.21 & 3 & 0.29 & 2 & 0.23 & 2 & 0.30 \\
\hline 75 & m7_ar3_1 & 1 & 0.52 & 2 & 0.58 & 4 & 0.37 & 2 & 0.63 & 2 & 0.60 \\
\hline 76 & m7_ar4_1 & 1 & 0.2 & 2 & 0.19 & 3 & 0.22 & 3 & 0.41 & 2 & 0.22 \\
\hline 77 & m7_ar5_1 & 1 & 0.15 & 2 & 0.29 & 4 & 0.35 & 2 & 0.61 & 2 & 0.33 \\
\hline 78 & no7_ar2_1 & 1 & 2.31 & 2 & 3.41 & 3 & 5.72 & 2 & 3.14 & 2 & 5.55 \\
\hline 79 & no7_ar25_1 & 2 & 6.3 & 4 & 16.59 & 5 & 21.21 & 3 & 10.35 & 4 & 17.64 \\
\hline 80 & no7_ar3_1 & 4 & 24.37 & 5 & 48.51 & 4 & 31.37 & 5 & 66.58 & 5 & 66.35 \\
\hline 81 & no7_ar4__1 & 4 & 15.81 & 6 & 65.98 & 6 & 38.25 & 6 & 72.78 & 6 & 73.61 \\
\hline 82 & no7_ar5_1 & 7 & 27.06 & 7 & 72.39 & 7 & 57.49 & 7 & 67.18 & 7 & 67.95 \\
\hline 83 & o7_2 & - & - & 6 & 280.21 & 5 & 157.73 & 5 & 223.75 & 6 & 268.26 \\
\hline 84 & o7_ar2_1 & 1 & 10.17 & 2 & 12.3 & 6 & 15.21 & 2 & 13.02 & 2 & 14.92 \\
\hline 85 & o7_ar25_1 & - & - & 5 & 139.07 & 6 & 90.03 & 5 & 125.5 & 5 & 208.92 \\
\hline 86 & o7_ar5_1 & - & - & 5 & 247.14 & 7 & 238.31 & 5 & 291.85 & 5 & 289.30 \\
\hline 87 & portfol_buyin & 7 & 0.09 & 6 & 0.11 & 6 & 0.1 & 5 & 0.12 & 5 & 0.13 \\
\hline 88 & portfol_card & 4 & 0.16 & 5 & 0.11 & 6 & 0.12 & 6 & 0.16 & 5 & 0.13 \\
\hline 89 & ravempb & - & - & - & - & - & - & - & - & - & - \\
\hline 90 & risk2bpb & 1 & 0.15 & 2 & 0.29 & 2 & 0.2 & 2 & 0.41 & 2 & 0.44 \\
\hline 91 & rsyn0805h & 1 & 0.11 & 2 & 0.17 & 3 & 0.18 & 3 & 0.34 & 2 & 0.21 \\
\hline 92 & rsyn0805m & 2 & 0.2 & 2 & 0.2 & 4 & 0.31 & 3 & 0.25 & 3 & 0.33 \\
\hline 93 & rsyn0805m02h & 5 & 0.54 & 4 & 0.75 & 4 & 0.53 & 3 & 0.67 & 3 & 0.53 \\
\hline 94 & rsyn0805m02m & 4 & 1.08 & 5 & 6.19 & 6 & 1.74 & 6 & 2.56 & 5 & 1.63 \\
\hline 95 & rsyn0805m03h & 3 & 0.55 & 4 & 1.13 & 3 & 0.8 & 3 & 0.98 & 3 & 0.88 \\
\hline 96 & rsyn0805m03m & 2 & 1.35 & 3 & 1.43 & 4 & 1.96 & 4 & 1.99 & 3 & 1.64 \\
\hline 97 & rsyn0805m04h & 2 & 0.53 & 3 & 1.45 & 3 & 1.54 & 2 & 1.3 & 2 & 1.39 \\
\hline 98 & rsyn0805m04m & 2 & 1.72 & 3 & 1.62 & 4 & 1.75 & 4 & 2.28 & 4 & 2.70 \\
\hline 99 & rsyn0810h & 1 & 0.11 & 2 & 0.16 & 2 & 0.18 & 3 & 0.32 & 2 & 0.18 \\
\hline 100 & rsyn0810m & 2 & 0.17 & 2 & 0.23 & 3 & 0.33 & 3 & 0.31 & 3 & 0.28 \\
\hline 101 & rsyn0810m02h & 3 & 0.43 & 3 & 2.48 & 3 & 0.7 & 4 & 2.56 & 4 & 2.66 \\
\hline 102 & rsyn0810m02m & 4 & 1.2 & 4 & 1.6 & 5 & 1.42 & 4 & 1.23 & 4 & 1.64 \\
\hline 103 & rsyn0810m03h & 3 & 0.78 & 3 & 1.28 & 4 & 1.2 & 3 & 1.37 & 4 & 1.48 \\
\hline 104 & rsyn0810m03m & 3 & 2.02 & 4 & 2.74 & 5 & 4.72 & 4 & 2.71 & 4 & 3.05 \\
\hline 105 & rsyn0810m04h & 3 & 0.9 & 3 & 1.3 & 4 & 1.68 & 4 & 1.69 & 3 & 1.55 \\
\hline 106 & rsyn0810m04m & 4 & 2.24 & 4 & 2.35 & 3 & 1.68 & 5 & 2.78 & 4 & 2.41 \\
\hline 107 & rsyn0815h & 1 & 0.14 & 2 & 0.28 & 3 & 0.24 & 3 & 0.41 & 2 & 0.28 \\
\hline 108 & rsyn0815m & 2 & 0.21 & 2 & 0.23 & 4 & 0.36 & 3 & 0.33 & 3 & 0.42 \\
\hline 109 & rsyn0815m02h & 3 & 0.44 & 4 & 0.94 & 3 & 0.74 & 3 & 0.86 & 2 & 0.63 \\
\hline 110 & rsyn0815m02m & 5 & 1.26 & 6 & 1.78 & 3 & 0.79 & 3 & 0.92 & 3 & 0.93 \\
\hline 111 & rsyn0815m03h & 5 & 1.12 & 5 & 2.2 & 4 & 1.36 & 4 & 1.98 & 3 & 1.64 \\
\hline 112 & rsyn0815m03m & 5 & 2.38 & 6 & 6.7 & 5 & 3.28 & 5 & 3.97 & 4 & 3.47 \\
\hline 113 & rsyn0815m04h & 3 & 1.04 & 4 & 2.47 & 3 & 1.7 & 3 & 2.06 & 2 & 1.71 \\
\hline 114 & rsyn0815m04m & 4 & 2.91 & 5 & 5.05 & 4 & 3.59 & 4 & 3.22 & 4 & 2.96 \\
\hline 115 & rsyn0820h & 3 & 0.23 & 3 & 0.38 & 3 & 0.33 & 5 & 0.44 & 5 & 0.43 \\
\hline 116 & rsyn0820m & 2 & 0.19 & 2 & 0.22 & 3 & 0.27 & 2 & 0.25 & 2 & 0.28 \\
\hline 117 & rsyn0820m02h & 3 & 0.46 & 3 & 0.87 & 3 & 0.88 & 6 & 1.24 & 6 & 1.31 \\
\hline 118 & rsyn0820m02m & 3 & 0.93 & 4 & 1.52 & 4 & 1.34 & 4 & 1.51 & 4 & 1.95 \\
\hline 119 & rsyn0820m03h & 2 & 0.69 & 3 & 1.49 & 4 & 2.79 & 4 & 1.64 & 3 & 1.74 \\
\hline 120 & rsyn0820m03m & 3 & 2.76 & 4 & 3.33 & 5 & 3.71 & 4 & 3.28 & 4 & 3.44 \\
\hline 121 & rsyn0820m04h & 4 & 1.68 & 4 & 3 & 4 & 2.95 & 3 & 2.39 & 4 & 2.45 \\
\hline 122 & rsyn0820m04m & 3 & 2.83 & 4 & 7.3 & 5 & 7.53 & 4 & 6.33 & 4 & 5.90 \\
\hline 123 & rsyn0830h & 4 & 0.3 & 4 & 0.46 & 4 & 0.48 & 5 & 0.82 & 4 & 0.48 \\
\hline 124 & rsyn0830m & 4 & 0.3 & 5 & 0.46 & 5 & 0.67 & 5 & 0.53 & 5 & 0.59 \\
\hline 125 & rsyn0830m03h & 2 & 0.7 & 3 & 2.06 & 4 & 3.49 & 3 & 2.15 & 3 & 2.42 \\
\hline 126 & rsyn0830m03m & 4 & 1.89 & 4 & 3.38 & 5 & 6.2 & 4 & 3.65 & 4 & 4 \\
\hline 127 & rsyn0830m04h & 3 & 1.44 & 3 & 2.95 & 4 & 3.29 & 3 & 3.03 & 3 & 3.19 \\
\hline 128 & rsyn0830m04m & 4 & 3.76 & 5 & 13.77 & 5 & 10.37 & 5 & 23.54 & 5 & 17.70 \\
\hline 129 & rsyn0840h & 2 & 0.21 & 3 & 0.38 & 4 & 0.43 & 3 & 0.45 & 3 & 0.48 \\
\hline 130 & rsyn0840m & 2 & 0.23 & 3 & 0.34 & 4 & 0.6 & 3 & 0.44 & 3 & 0.55 \\
\hline 131 & rsyn0840m03m & 4 & 1.94 & 5 & 4.57 & 4 & 2.38 & 5 & 4.63 & 5 & 4.86 \\
\hline 132 & rsyn0840m04h & 2 & 1.42 & 3 & 3.9 & 3 & 5.65 & 3 & 4.15 & 4 & 4.91 \\
\hline 133 & rsyn $0840 \mathrm{~m} 04 \mathrm{~m}$ & 4 & 3.81 & 5 & 17.03 & 4 & 7.43 & 5 & 10.07 & 5 & 10.36 \\
\hline 134 & smallinvDAXr1b010-011 & 43 & 2.88 & 38 & 2.7 & 37 & 2.59 & 18 & 1.18 & 18 & 1.30 \\
\hline 135 & smallinvDAXr1b020-022 & 44 & 6.25 & 46 & 3.42 & 42 & 2.63 & 15 & 1.31 & 32 & 2.74 \\
\hline 136 & smallinvDAXr1b050-055 & 67 & 6.98 & 70 & 3.45 & 62 & 3.5 & 27 & 1.69 & 29 & 2.05 \\
\hline 137 & smallinvDAXr1b100-110 & 92 & 9.16 & 88 & 3.78 & 95 & 4.3 & 37 & 2.05 & 26 & 1.21 \\
\hline 138 & smallinvDAXr1b150-165 & 103 & 9.26 & 101 & 4.64 & 119 & 5.26 & 23 & 1.24 & 28 & 1.29 \\
\hline 139 & smallinvDAXr1b200-220 & 111 & 24.56 & - & - & 115 & 4.67 & 22 & 1.21 & 20 & 0.89 \\
\hline 140 & smallinvDAXr2b010-011 & 43 & 2.9 & 35 & 2.18 & 37 & 2.75 & 18 & 0.92 & 20 & 1.68 \\
\hline 141 & smallinvDAXr2b020-022 & 48 & 5.75 & 42 & 3.35 & 48 & 2.97 & 19 & 1.4 & 32 & 2.39 \\
\hline 142 & smallinvDAXr2b050-055 & 70 & 6.29 & 68 & 3.63 & 67 & 3.72 & 29 & 1.99 & 31 & 2.11 \\
\hline 143 & smallinvDAXr2b100-110 & 92 & 8.38 & 93 & 4.04 & 103 & 4.5 & 37 & 1.99 & 20 & 1.01 \\
\hline 144 & smallinvDAXr2b150-165 & 104 & 10.6 & 93 & 4.3 & 76 & 3.34 & 48 & 2.52 & 25 & 1.19 \\
\hline 145 & smallinvDAXr2b200-220 & 97 & 20.44 & 116 & 4.79 & 126 & 5.73 & 25 & 4.33 & 27 & 1.29 \\
\hline 146 & smallinvDAXr3b010-011 & 43 & 3.99 & 38 & 2.63 & 40 & 2.62 & 18 & 1.16 & 20 & 1.05 \\
\hline 147 & smallinvDAXr3b020-022 & 48 & 5.94 & 39 & 2.88 & 38 & 2.71 & 19 & 1.34 & 30 & 2.53 \\
\hline 148 & smallinvDAXr3b050-055 & 67 & 6.69 & 65 & 3.77 & 77 & 3.88 & 26 & 1.95 & 31 & 2.15 \\
\hline
\end{tabular}




\begin{tabular}{|c|c|c|c|c|c|c|c|c|c|c|c|}
\hline \multicolumn{2}{|r|}{ Examples } & \multicolumn{2}{|c|}{ Bonmin } & \multicolumn{2}{|c|}{$\mathrm{OA}$} & \multicolumn{2}{|c|}{$\mathrm{OA}+\mathrm{FP}$} & $\mathrm{POA}$ & $1,1 / 3)$ & POA & $0,1 / 3)$ \\
\hline Id & Name & \#It. & Time & \#It. & Time & \#It. & Time & \#It. & Time & \#It. & Time \\
\hline 150 & smallinvDAXr3b150-165 & 104 & 10.47 & 94 & 4.43 & 113 & 5.3 & 46 & 2.73 & 30 & 1.58 \\
\hline 152 & smallinvDAXr4b010-011 & 43 & 3.74 & 39 & 2.88 & 40 & 2.96 & 19 & 1.07 & 20 & 1.61 \\
\hline 153 & smallinvDAXr4b020-022 & 50 & 7.31 & 44 & 3.14 & 35 & 2.82 & 17 & 1.33 & 28 & 2.12 \\
\hline 154 & smallinvDAXr4b050-055 & 66 & 6.54 & 73 & 3.79 & 70 & 3.72 & 26 & 2.51 & 31 & 2.21 \\
\hline 157 & smallinvDAXr4b200-220 & 113 & 25.51 & 129 & 5.82 & 101 & 4.19 & 27 & 1.59 & 28 & 1.38 \\
\hline 158 & smallinvDAXr5b010-011 & 41 & 3.02 & 37 & 2.62 & 39 & 2.65 & 17 & 1.06 & 20 & 1.28 \\
\hline 159 & smallinvDAXr5b020-022 & 46 & 4.89 & 40 & 2.66 & 39 & 2.95 & 18 & 1.73 & 24 & 1.54 \\
\hline 160 & smallinvDAXr5b050-055 & 62 & 5.86 & 68 & 4.22 & 77 & 4.3 & 28 & 1.81 & 29 & 2.01 \\
\hline 161 & smallinvDAXr5b100-110 & 82 & 8.63 & 99 & 4.66 & 82 & 3.48 & 41 & 2.67 & 25 & 1.91 \\
\hline 162 & smallinvDAXr5b150-165 & 114 & 12.07 & 99 & 4.5 & 106 & 4.78 & 27 & 1.42 & 22 & 1.15 \\
\hline 167 & sssd15-06 & 11 & 13.71 & 11 & 28.94 & 9 & 9.69 & 9 & 7.82 & 9 & 75.55 \\
\hline 168 & sssd15-08 & 15 & 19.71 & 11 & 205.1 & 8 & 100.55 & 7 & 83.58 & - & - \\
\hline 169 & sssd $16-07$ & 16 & 27.77 & 13 & 142.49 & 10 & 45.75 & 10 & 36.64 & 9 & 50.72 \\
\hline 170 & sssd18-06 & 11 & 11.54 & 10 & 11.93 & 10 & 31.03 & 9 & 7.39 & 8 & 31.01 \\
\hline 171 & sssd20-04 & 10 & 4.42 & 9 & 5.14 & 9 & 2.08 & 8 & 1.61 & 8 & 1.73 \\
\hline 172 & sssd25-04 & 9 & 2.73 & 6 & 2.76 & 13 & 14.17 & 8 & 1.62 & 9 & 4.46 \\
\hline 173 & st_e14 & 3 & 0.05 & 3 & 0.09 & 3 & 0.07 & 4 & 0.22 & 3 & 0.12 \\
\hline 174 & syn05h & 2 & 0.04 & 2 & 0.04 & 2 & 0.04 & 2 & 0.06 & 2 & 0.05 \\
\hline 175 & syn05hfsg & 2 & 0.04 & 2 & 0.04 & 2 & 0.04 & 2 & 0.05 & 2 & 0.06 \\
\hline 176 & syn05m & 2 & 0.03 & 2 & 0.05 & 2 & 0.04 & 2 & 0.05 & 2 & 0.05 \\
\hline 177 & syn $05 \mathrm{~m} 02 \mathrm{~h}$ & 1 & 0.04 & 1 & 0.04 & 1 & 0.05 & 1 & 0.06 & 1 & 0.06 \\
\hline 178 & syn05m02hfsg & 1 & 0.05 & 1 & 0.04 & 1 & 0.05 & 1 & 0.06 & 1 & 0.06 \\
\hline 187 & syn $10 \mathrm{~m}$ & 2 & 0.04 & 2 & 0.05 & 2 & 0.04 & 2 & 0.07 & 2 & 0.06 \\
\hline 188 & syn10m02h & 1 & 0.06 & 2 & 0.1 & 2 & 0.12 & 2 & 0.14 & 2 & 0.15 \\
\hline 189 & syn10m02hfsg & 1 & 0.17 & 2 & 0.13 & 2 & 0.12 & 2 & 0.14 & 2 & 0.15 \\
\hline 190 & syn $10 \mathrm{~m} 02 \mathrm{~m}$ & 2 & 0.07 & 2 & 0.11 & 2 & 0.11 & 2 & 0.16 & 2 & 0.17 \\
\hline 191 & syn10m03h & 1 & 0.09 & 2 & 0.15 & 2 & 0.24 & 2 & 0.22 & 2 & 0.21 \\
\hline 192 & syn $10 \mathrm{~m} 03 \mathrm{~m}$ & 1 & 0.06 & 2 & 0.16 & 2 & 0.21 & 2 & 0.21 & 2 & 0.19 \\
\hline 193 & syn $10 \mathrm{~m} 04 \mathrm{~h}$ & 1 & 0.09 & 2 & 0.18 & 3 & 0.26 & 3 & 0.4 & 2 & 0.21 \\
\hline 194 & syn $10 \mathrm{~m} 04 \mathrm{~m}$ & 1 & 0.08 & 2 & 0.23 & 2 & 0.21 & 2 & 0.28 & 2 & 0.33 \\
\hline 195 & syn $15 \mathrm{~h}$ & 1 & 0.05 & 1 & 0.06 & 1 & 0.08 & 1 & 0.08 & 1 & 0.09 \\
\hline 196 & syn15hfsg & 1 & 0.04 & 1 & 0.07 & 1 & 0.08 & 1 & 0.08 & 1 & 0.08 \\
\hline 197 & syn $15 \mathrm{~m}$ & 2 & 0.05 & 2 & 0.07 & 2 & 0.08 & 2 & 0.1 & 2 & 0.10 \\
\hline 198 & syn $15 \mathrm{~m} 02 \mathrm{~h}$ & 1 & 0.07 & 2 & 0.16 & 2 & 0.14 & 2 & 0.21 & 2 & 0.20 \\
\hline 199 & syn $15 \mathrm{~m} 02 \mathrm{~m}$ & 1 & 0.06 & 2 & 0.33 & 3 & 0.19 & 3 & 0.42 & 2 & 0.36 \\
\hline 200 & syn $15 \mathrm{~m} 03 \mathrm{~h}$ & 1 & 0.09 & 2 & 0.23 & 2 & 0.21 & 3 & 0.32 & 3 & 0.32 \\
\hline 201 & syn $15 \mathrm{~m} 03 \mathrm{~m}$ & 2 & 0.11 & 2 & 0.21 & 3 & 0.24 & 3 & 0.95 & 2 & 0.84 \\
\hline 202 & syn $15 \mathrm{~m} 04 \mathrm{~h}$ & 1 & 0.12 & 2 & 0.29 & 3 & 0.4 & 10 & 0.58 & 3 & 0.38 \\
\hline 203 & syn $15 \mathrm{~m} 04 \mathrm{~m}$ & 2 & 0.13 & 3 & 0.47 & 3 & 0.43 & 4 & 0.71 & 3 & 0.54 \\
\hline 204 & syn $20 \mathrm{~h}$ & 2 & 0.08 & 2 & 0.1 & 2 & 0.12 & 5 & 0.3 & 2 & 0.11 \\
\hline 205 & syn20hfsg & 2 & 0.08 & 2 & 0.09 & 2 & 0.11 & 5 & 0.3 & 2 & 0.11 \\
\hline 206 & syn $20 \mathrm{~m}$ & 2 & 0.06 & 2 & 0.07 & 2 & 0.1 & 2 & 0.12 & 2 & 0.13 \\
\hline 207 & syn $20 \mathrm{~m} 02 \mathrm{~h}$ & 2 & 0.13 & 2 & 0.21 & 2 & 0.21 & 2 & 0.24 & 2 & 0.25 \\
\hline 208 & syn $20 \mathrm{~m} 02 \mathrm{~m}$ & 2 & 0.09 & 3 & 0.71 & 3 & 0.19 & 3 & 0.68 & 3 & 0.69 \\
\hline 209 & syn $20 \mathrm{~m} 03 \mathrm{~h}$ & 1 & 0.12 & 2 & 0.29 & 3 & 0.39 & 3 & 0.63 & 2 & 0.36 \\
\hline 210 & syn $20 \mathrm{~m} 03 \mathrm{~m}$ & 2 & 0.14 & 3 & 0.36 & 3 & 0.33 & 3 & 0.45 & 3 & 0.50 \\
\hline
\end{tabular}




\section{References}

[1] M. A. Duran and I. E. Grossmann. An outer-approximation algorithm for a class of mixed-integer nonlinear programs. Mathematical Programming, 36(3):307-339, 1986.

[2] I. Quesada and I.E. Grossmann. An LP/NLP based branch and bound algorithm for convex MINLP optimization problems Computers \&3 Chemical Engineering, 16(10-11):937-947, 1992.

[3] R. Fletcher, S. Ley, and S. Leyffer. Solving mixed integer nonlinear programs by outer approximation. Mathematical Programming, 66(1-3):327-349, 1994.

[4] S. Leyffer. Generalized outer approximation. Encyclopedia of Optimization, 2(1):247-254, 2001.

[5] J. Kronqvist, D. E. Bernal, and I. E. Grossmann. Using regularization and second order information in outer approximation for convex MINLP Mathematical Programming, 2018.

[6] H. Hijazi, P. Bonami, and A. Ouorou. An outer-inner approximation for separable mixed-integer nonlinear programs. INFORMS Journal on Computing, 26(1):31-44, 2014.

[7] J. Kronqvist, A. Lundell, and T. Westerlund. The extended supporting hyperplane algorithm for convex mixed-integer nonlinear programming. Journal of Global Optimization, 64(2):249-272, 2016.

[8] M. Hunting. The AIMMS outer approximation algorithm for MINLP. Technical Report AIMMS B.V., 2011.

[9] Lundell, A. Kronqvist, J. and Westerlund, T. The Supporting Hyperplane Optimization Toolkit: A Polyhedral Outer Approximation Based Convex, 2018.

[10] J. Kronqvist, D. E. Bernal, A. Lundell, and I. E. Grossmann. A review and comparison of solvers for convex MINLP Optimization and Engineering, 20(2):397-455, 2019.

[11] J. Kronqvist, D. E. Bernal, A. Lundell and T. Westerlund. A center-cut algorithm for quickly obtaining feasible solutions and solving convex MINLP problems Computers \&3 Chemical Engineering, 122, 105-113, 2019 .

[12] I. E. Grossman. Review of nonlinear mixed-integer and disjunctive programming techniques. Optimization and Engineering, 3(3):227-252, 2002.

[13] S. Leyffer. Deterministic Methods for Mixed Integer Nonlinear Programming. PhD thesis, 1993.

[14] M. Fischetti, F. Glover, and A. Lodi. The feasibility pump. Mathematical Programming, 104(1):91-104, 2005.

[15] P. Bonami, G. Cornuéjols, A. Lodi, and F. Margot. A feasibility pump for mixed integer nonlinear programming. Mathematical Programming, 119(2):331-352, 2009.

[16] C. D'Ambrosio. Application-oriented Mixed Integer Non-Linear Programming. PhD thesis, 2009.

[17] B. Geißler, A. Morsi, L. Schewe and M. Schmidt. Penalty Alternating Direction Methods for Mixed-Integer Optimization: A New View on Feasibility Pumps. SIAM Journal on Optimization, 27(3):1611-1636, 2017.

[18] P. Bonami and J. P. M. Gonçalves. Heuristics for convex mixed integer nonlinear programs. Computational Optimization and Applications, 51(2):729-747, 2012.

[19] T. Berthold. Primal MINLP Heuristics in a Nutshell. Operations Research Proceedings 2013, 481-486, 2013.

[20] M. Fischetti and D. Salvagnin. Feasibility pump 2.0. Mathematical Programming Computation, 1(2-3):201$222,2009$.

[21] T. Achterberg, T. Berthold. Improving the feasibility pump. Discrete Optimization, 4(1):77-86, 2007.

[22] M. R. Bussieck and S. Vigerske. MINLP Solver Software. In Wiley Encyclopedia of Operations Research and Management Science, pages 1-17. 2011.

[23] T. Berthold and J. M. Sullivan. Heuristic algorithms in global MINLP solvers. PhD thesis, 2014.

[24] M. De Santis, S. Lucidi and F. Rinaldi. Feasibility Pump-like heuristics for mixed integer problems. Discrete Applied Mathematics, 165:152-167, 2014.

[25] S. Sharma, B. R. Knudsen, and B. Grimstad. Towards an objective feasibility pump for convex MINLPs. Computational Optimization and Applications, 63(3):737-753, 2016.

[26] Berthold, T. RENS: The optimal rounding. Mathematical Programming Computation, 6(1):33-54. 2014. 
[27] C. DAmbrosio, A. Frangioni, L. Liberti, and A. Lodi. A storm of feasibility pumps for nonconvex MINLP. Mathematical Programming, 136(2):375-402, 2012.

[28] M. Fischetti and M. Monaci. Proximity search for 0-1 mixed-integer convex programming. Journal of Heuristics, 20(6):709-731, 2014.

[29] D. E. Bernal, S. Vigerske, F. Trespalacios, and I. E. Grossmann. Improving the performance of DICOPT in convex MINLP problems using a feasibility pump. 2017.

[30] M. De Santis, S. Lucidi and F. Rinaldi. A New Class of Functions for Measuring Solution Integrality in the Feasibility Pump Approach. SIAM Journal on Optimization, 23(3):1575-1606, 2013.

[31] A. Wächter and L. T. Biegler. On the Implementation of a Primal-Dual Interior Point Filter Line Search Algorithm for Large-Scale Nonlinear Programming Mathematical Programming, 106(1):25-57, 2006. Homepage: "https://projects.coin-or.org/Ipopt"

[32] P. Bonami, L. T. Biegler, A. R. Conn, G. Cornujols, I. E. Grossmann, C. D. Laird, A. Wächter et al. An algorithmic framework for convex mixed integer nonlinear programs. Discrete Optimization, 5(2):186-204, 2008. Homepage: "https://projects.coin-or.org/Bonmin"

[33] Cplex homepage: "https://www.ibm.com/analytics/cplex-optimizer"

[34] MINLPLib: "http://www.minlplib.org" 